\title{
Countering Disruptive Innovations
}

\author{
Boniface Okanga \\ Edinburgh Napier University \\ Scotland
}

\begin{abstract}
Countering disruptive innovations is critical for bolstering a business' continuity and sustainability. Unfortunately, as theories on how to counter disruptive innovations remain largely underdeveloped, the identification of meaningful business models that can be replicated to counter disruptive innovations also seems a challenge that still confounds most of the contemporary business executives. It is such a challenge that this research sought to address by using a meta-synthesis to analyse the strategic management and marketing theories to identify a combination of strategies that can constitute a business model that the executives can replicate to counter disruptive innovations. To counter disruptive innovations, findings revealed proactive strategic analysis and sensing to be of essence for identifying unfilled low-end or new-market footholds that can be filled through new value innovations before disrupters are able to do so. It is further posited that such initiatives must be accompanied with product modifications to enrich the features, quality, designs and functionality of the existing products to counter the disrupters' gradual creep into the incumbents' mainstream up-markets. Besides assimilation of disrupters' unique technologies and competencies through acquisitions and strategic alliance and partnership with disrupters, incumbents can also pursue organic assimilation of disrupters' unique technologies and core competencies through extensive investments in R\&D. The paper concludes with the suggestion of a business model that businesses can replicate to counter disruptive innovations.
\end{abstract}

Keywords: Disruptive Innovations, Low-end footholds, Market Performance, New-Market footholds, New Value Innovations, Superior Products.

\section{Introduction}

Countering disruptive innovations is a prerequisite for leveraging a business' sustainability. In the midst of the increasing proliferations of the different forms of the modern disruptive innovations, the use of appropriate proactive strategies disrupts the would have been disrupters to bolster a business' continuity. Disruptive innovations are new products or business models that use low-end or new-market footholds as paths to access and attract more and more customers from a firm's mainstream market segments (Christensen, Raynor \& McDonald, 2015). If this gradual creep into the upmarket is not disrupted by the incumbents, disruptive innovations can cause disruptive effects on the incumbents' market performance. Disruptive innovations which are undisrupted by the incumbents or unconstrained by momentum, tech-implementation, ecosystem, new technology and business model barriers can cause significant erosion of the incumbents' mainstream market. As this affects the incumbent's sales, revenues, profits and returns on shareholders' value, it can also cause the incumbent's complete market exit (Weeks, 2015). In effect, proactive strategic analysis and sensing of the likelihood of the emergence of disruptive innovations are critical for identifying and countering disruptive innovations before they become disruptive. However, as theories on how to counter disruptive innovations remain largely underdeveloped, the identification of meaningful business models that can be replicated to counter disruptive innovations also seems to remain a challenge that most of 


\section{Boniface Okanga \\ Countering Disruptive Innovations}

the contemporary business executives still grapple with. This research seeks to address this challenge by undertaking relevant analysis of the strategic management and marketing theories to identify a combination of strategies that can constitute a business model that the executives can replicate to counter disruptive innovations.

\subsection{Problem Statement}

Countering disruptive innovations to leverage a business' continuity and sustainability is a paradox that confounds most of the contemporary business executives.

\subsection{Aim of the study}

The purpose of this research is to develop a business model for countering disruptive innovations in the increasingly competitive modern business environment.

\section{Literature Review}

Disruptive innovation is not an event, but a process that unfolds overtime from low-end or new-market foothold upstarts to the incumbents' mainstream markets. It refers to the process of creating superior products that are offered at relatively lower prices to target market segments that are presently ignored or underserved by the existing incumbents (Christensen et al. 2015). Quite often, such a process arises from the fact that as incumbent businesses focus on devising strategies to respond to the most demanding profitable market segments, they tend to ignore or underserve the less demanding market segments that are also usually considered as less profitable. It is this strategic business approach of the incumbents that create gaps that disrupters utilise to create products that respond to the needs of such underserved market segments. As disrupters target such market segments, they continue to gradually build capabilities to not only provide superior value offerings, but also capabilities to target more customers from such segments.

However, since the target of the incumbents is usually the most profitable market segments, most incumbents often do not react vigorously to disrupter's activities. This is because without thorough analysis, the likely devastating effects of most disruptive innovations are often not easily discernible in their early stages of market entrance. Yet as incumbents fail to detect disrupters, it offers even greater opportunities for disrupters to build further capabilities to not only target customers in the lower-end market segments, but also the mainstream customers that are considered as the most profitable by the incumbents. As they undertake such initiatives, they tend to preserve advantages such as cost control and capabilities to offer superior qualities that influenced their initial success (Raynor \& Mumtaz, 2013). The implications of these are often latent in the fact that disruption tends to occur when a significant number of the customers in the incumbents' most profitable segments begin to embrace the disrupter's offerings. At such points, Figure 1 signifies it is often too late for the incumbents to successfully retaliate. In case of the incumbents' retaliations, most forms of retaliations often entail restructuring, downsizing and disinvestments that in most of the cases cause more disruptions (Christensen, 1995). As this affects the incumbents' market performance, it also causes their probable exit. 


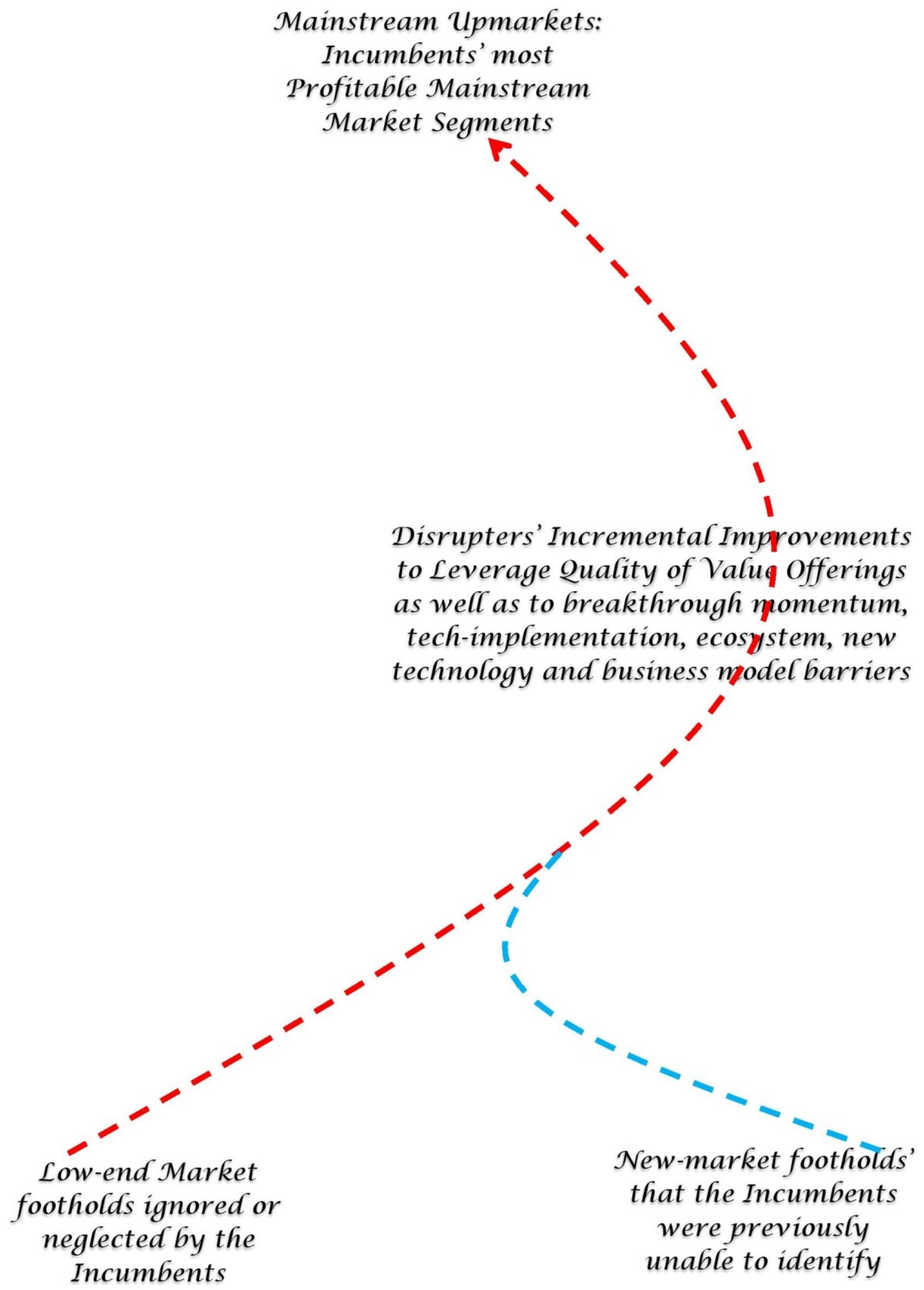

Figure 1: The Origin and Creep of Disruptive Innovations into the Incumbents' Mainstream upmarkets

However, not all innovations are disruptive. Innovation can be sustaining or radically new and disruptive. Sustaining innovations often focus on modifying the features, designs and 


\section{Boniface Okanga \\ Countering Disruptive Innovations}

functionality of the existing products to leverage a firm's performance in the existing markets. Due to the direct confrontations that they instigate, sustaining innovations are often susceptible to aggressive devastating retaliations from rivals. In effect, sustaining innovations are often thwarted before they become disruptive. In contrast, disruptive innovations as illustrated in Figure 1, originate from low-end or new market footholds. Whereas in low-end footholds, disrupters focus on the underserved less demanding and less profitable customer segments, in new-market footholds, disrupters create new markets that never existed by converting non-consumers into customers (Christensen, 1997).

Yet, as disrupters target customers in low-end or new market footholds, mainstream customers are often not their targets. Instead, mainstream customers only emerge as the focus of disrupters when continuous cost and quality incremental improvements undertaken by disrupters elevate the quality of the disrupters' innovations to match or even exceed the expectations of most of the customers in the incumbents' mainstream markets. This signifies disrupters usually avoid direct confrontations with the incumbents by directly targeting mainstream customers during the initial stages of their market entrance (Christensen et al. 2015). This is attributable to the fact that attempts to do so would certainly instigate aggressive reactions from the incumbents to disrupt disrupters before they become disruptive to the incumbents.

In effect, disruptive innovation is not an event, but a process that unfolds overtime from low-end or new-market foothold upstarts to the incumbents' mainstream markets. Due to these lengthy processes, disruptive innovations are often ignored by the incumbents because in some of the cases they fail and rise again as they make gradual movements towards the incumbents' mainstream markets. Disruptive innovations are also often ignored by most incumbents because they initially never target the incumbents' mainstream markets. To distract incumbents' attention, disrupters often develop and build models that are at odds with the models being used by the incumbents (Christensen et al. 2015).

However, when disruptive innovations subsequently begin to erode the incumbents' mainstream markets, their impacts may tend to be quite devastating. In such cases, disruptive innovations may not only cause declining sales, revenues, profitability and returns on shareholders' value, but also a firm's probable exit. That implies constant analysis and devising of the strategies for dealing with disruptive innovations are prerequisites for leveraging a business' overall sustainability (Raynor \& Mumtaz, 2013). Unfortunately, research and development of the theories for dealing with disruptive innovations remain largely premature for businesses to identify any meaningful business models that can be replicated to counter threats from disruptive innovations. It is such theoretical gap that this research deals with.

\section{Research Methodology}

To reach logical conclusions on a business model that can be developed for countering disruptive innovations, the study used interpretivist research paradigm to undertake critical content analysis of the existing strategic management and marketing theories. The motive of such analysis was to discern and extract a combination of strategies that can constitute a business model that can be utilised for countering disruptive innovations. To accomplish that, the study undertakes a meta-synthesis of strategic management theories to assess what disruptive innovations are or are not. Some of the theories analysed in this initiative were Christensen, Raynor and McDonald's (2015) "What is Disruptive Innovations?", Christensen's (1995) "Disruptive Technologies", Christensen's (1997) "Innovator's Dilemma", Weeks' (2015) "Disruption Theory" and Assink's (2006) "Inhibitors of Disruptive Innovation Capability". Understanding the definition of disruptive innovations created the theoretical foundation for further critical analysis of the strategic management and marketing theories 


\section{Boniface Okanga \\ Countering Disruptive Innovations}

to identify a combination of the strategies that could be used for countering disruptive innovations.

The strategic management theories examined included Reeves and Deimler's (2011) "Adaptability" theory where the concept of strategic analysis and sensing was found to be critical for leveraging identification and thwarting of disrupters in their early stages. This was followed by analysis of Kim and Mauborgne's (2005) "Blue Ocean Strategy" where embracement of a culture of new value innovations emerged as critical for aiding incumbents identify and fill the unfilled market gaps before disrupters are able to do so. In strategic marketing theories, Yannopoulos' (2011) "Defensive and Offensive Strategies for Market Success" revealed some of the critical strategies that can be replicated to counter disruptive innovations to include introduction of fighting brands, covering all bases and continuous improvement.

Wessel and Christensen's (2012) theory for "Surviving Disruption" herald the essence for the analysis and identification of the disrupter's business model vis-à-vis a firm's own business model as well as the associated probable barriers as some of the essential strategies for discerning the disrupters' potential to complete the disruptions. As on the otherhand, the interpretation of Grant's (2010) "Contemporary Strategic Management" highlighted mergers and acquisitions, strategic alliance and partnership as well as extensive investments in R\&D as strategies that aid assimilation of disrupters' unique technologies and capabilities to counter the disruptive effects of disruptive innovations. As indicated in the sections that follow, this analysis influenced the identification and formulation of a business model that would leverage a business' capabilities to effectively counter disruptive innovations.

\section{Data Analysis And Interpretation}

Outcomes of meta-synthesis imply countering disruptive innovations requires not only proactive strategic analysis and sensing of disruptive innovations, but also leverage of capabilities that constrain disrupters to spawn retention of the incumbents' mainstream customers (Kim \& Mauborgne, 2005; Reeves \& Deimler, 2011: Wessel \& Christensen, 2012).

\subsection{Proactive Strategic Analysis and Sensing of Disruptive Innovations}

Proactive strategic analysis and sensing would aid identification and thwarting disrupters before they become devastatingly disruptive (Reeves \& Deimler, 2011). However, such analysis must not only focus on identifying or sensing probable disrupters, but also unfilled gaps in the market. Unfilled gaps in the market may arise either from the customers that are completely ignored or underserved (Reeves \& Deimler, 2011). It may also arise from certain aspects of the existing product's usage or functionalities that are presently not responsive to the needs and demands of the existing customers. The identification of these unfilled gaps must be accompanied with new value innovations to fill such gaps before prospective disrupters are able to utilise them (Kim \& Mauborgne, 2005).

However, risks of such a strategy may arise from the scalability of the usually insufficient financial resources to produce multiple product lines to fill all gaps. It may also induce overexpansion that instead constrains concentration of the executives on the areas of significant importance. This may subsequently expose other business weaknesses that can be utilised by the competitors to attack and undermine a firm's performance. Nevertheless, even if such a strategy is inherently ingrained with certain weaknesses, its usage still places a firm in a better position as compared to when it has to deal with the risks of being shut down by disrupters. Yet, as a firm undertakes new value innovations to fill the unfilled gaps in the market, increased investment in R\&D may also catalyse the capabilities of the firm to introduce fighting brands that target disrupters (Yannopoulos 2011). This would disrupt 


\section{Boniface Okanga \\ Countering Disruptive Innovations}

disrupters to minimise the magnitude of the devastating effects of their actions on a business' performance.

The introduction of fighting brands is often accomplished by innovation of a brand priced lower than a firm's premium brand so that as the fighting brand undermines the performance of disrupters, a firm's core premium brands are usually not affected. However, since disrupters often not only focus on pricing, but also quality and functionality of the products, it is often of essence that fighting brands are not only cheaper, but also of superior quality and functionality (Yannopoulos 2011). Investment in superior product quality and functionality may however cause cost escalations to suggest that even if a firm incurs losses, some form of predatory engagement is still critical for driving disrupters out of the market. Such initiatives must be accompanied by continuous improvement to spawn the improvement of the quality, designs, features and functionality of the existing products.

Continuous improvement must also encompass discerning how to improve production efficiency and cost management to lower the costs and prices of the existing products (Hekkert \& Simona, 2011). The application of such a strategy would enable a firm respond more to the gaps that the disrupters are targeting to facilitate their movements towards more and more customers in the upstream market. Even in the midst of the application of such strategies, some of the strategies for dealing with disruptive innovations may still require either direct acquisition of the disrupters or the assimilation of their technologies and competencies by headhunting of the personnel from the emerging disruptive businesses (Grant, 2010).

In such instances, strategic alliances and partnerships may also be formed with the likely future disrupters. This would enable a firm assimilate and integrate new technologies and competencies that are critical for them to counter threats from disruptive innovations (Grant, 2010). However, if it only emerges to be too late for the executives to proactively identify and curtail the emergence of new disruptive businesses, the executives may instead have to identify and capitalise on the capabilities that disrupters do not possess to thwart their creep into the mainstream markets.

\subsection{Leveraging Capabilities and Advantages that Constrain Disrupters' Creep into the Incumbents' Mainstream Markets}

Leveraging capabilities and advantages that constrain disrupters' creep into the incumbents' mainstream markets is one of the strategies for dealing with disruptive innovations. This is attributable to the fact that just like any other businesses, disrupters also often face certain capabilities' constraints that hamper the speed and fastness of their disruptions. Hence, it is critical for incumbents to identify such disadvantages and use them to thwart the disrupters' creep into the main stream markets. To accomplish this, Wessel and Christensen (2012) suggest the essence for the analysis and identification of the disrupter's business model visà-vis a firm's own business model. Such analysis must be accompanied by the evaluation of the probable barriers that would hinder the disrupter's capabilities to co-opt a firm's current advantages in the future.

The analysis and identification of the disrupter's business model vis-à-vis a firm's own business model must entail the evaluation of the disrupter's extendable core. Extendable core constitutes of the capabilities that would enable disrupters maintain or even improve their existing performance advantages whilst also creeping into the upmarket to tap more and more of the incumbent's more profitable market segments (Wessel \& Christensen, 2012). This extendable core is explained by the disrupter's cost management and quality management capabilities as well as unique technologies and core competencies to continue offering products with superior functionalities. The implications are latent in the fact that if disrupters are found to possess enormous advantages and fewer disadvantages in the 


\section{Boniface Okanga \\ Countering Disruptive Innovations}

accomplishment of similar business tasks to serve the same market, then, the disruption would be faster and complete. This contrasts with the situation where disrupters face disadvantages or certain barriers towards the accomplishment of similar tasks to serve the same market segments. Some of the barriers that hinder disrupters' creep into the mainstream upmarkets often arise from momentum, tech-implementation, ecosystem, new technology and business model barriers.

Whereas momentum barriers arise from the circumstances where customers are found to be more used to the status quo, tech-implementation barriers often emanate from the situations where threats from the disrupters could be over-come using the existing technologies. Ecosystem barriers arise if change in business environment is required for disrupters to complete the disruption. This contrasts with new technology barriers that often emerge from lack of the technology needed to change the competitive business landscape, and the business model barrier that would require disrupters to adopt similar cost structure to complete the process of disruption (Assink, 2006). The analysis and identification of some of these barriers are important for identifying the advantages and competencies that incumbents must continue to build and improve to retain most of their profitable mainstream customers.

The application of such a strategy enables incumbents thwart the growth of disrupters into the mainstream market. This view is echoed in the fact that some of these barriers, especially the technology barriers explain why despite the invention of large airship, the disruption of the large ocean cargo ship industry is yet incomplete due to fact that new technology does not yet exist to facilitate the transportation of extremely heavy goods by aeroplanes. As in the grocery retail industry, the disruption of the grocery retail industry is yet incomplete due to the inability of the disrupters to disrupt brick-and-mortar shopping as leisure and an outdoor entertainment activity.

In South Africa, similar trends are also evident in the telecommunication industry on the basis that although the emergence of cellular networks such as MTN, Vodacom and Cell " $\mathrm{C}$ " disrupted Telkom's fixed telephone lines, the disruption is yet incomplete due to the inabilities of MTN, Vodacom and Cell "C" as major disrupters to overcome technological barriers that limit the provision of lower internet connection costs. The implications are latent in the fact that as MTN, Vodacom and Cell " $\mathrm{C}$ " continue to grapple with how to integrate the provision of more efficient and low cost internet connections as part of their strategic business units, Telkom has been using such advantages to retain most of their profitable mainstream customers. Similar trends are also evident in East Africa where the emergence of mobile money transfer as a disruptive business model did not complete the disruption of the legacy banking systems.

Mobile money transfer uses cellular network technology to facilitate the transfer of money as well as payment of salaries and other monetary obligations. Although this disrupted the traditional retail banking, the disruption was incomplete on the basis that mobile money has not been able to creep into the core banking business transactions of accepting and keeping deposits, as well as offering insurance, financial advice and credit facilities. This is not only attributable to the ecosystem barriers that would require change and transformation of the existing business environment for mobile money to complete its disruption, but also technological and business model barriers that would require mobile money to adopt similar technologies and cost structure to complete the process of disruption (Durantin, Fanmuy, Miet \& Pegon, 2017). In otherwords, this demonstrates how capabilities that constrain disrupters can be leveraged to spawn retention of the incumbents' mainstream customers as one of the strategies for dealing with disruptive innovations. 


\section{Conclusion And Recommendations}

Disruptive innovations are most business' nightmares in the $21^{\text {st }}$ Century. As disruptive innovations creep through the low-end or new-market footholds into the incumbent's mainstream upmarkets, they disrupt and interfere with a firm's growth potential. This is attributable to the fact that due to unique technologies and business models that are often at odds with the incumbent's technologies and business models, disrupters are often able to undertake undisrupted incremental improvements. These incremental improvement initiatives enable disrupters leverage the superiority of the quality of their value offerings to a level that cannot easily be matched by incumbents. Combined with cost management capabilities that initially drove their success in the original low-end or new-market footholds, all these offer disrupters with capabilities to offer superior value offerings at relatively lower prices. It is these superior advantages over incumbents that sustain disrupters' capabilities to continuously attack and erode incumbents' market share without much disruptions from the incumbents. Yet, as disrupters make significant inroads into the incumbents' mainstream upmarkets; further devastating disruptive effects are often latent in declining sales, profitability and subsequently a firm's exit from the market. To minimise risks of vulnerability to disruptive innovations, theoretical analysis and interpretation suggest countering disruptive innovations would require not only proactive strategic analysis and sensing of disruptive innovations, but also leverage of capabilities that constrain disrupters to spawn retention of the incumbents' mainstream customers.

Unfortunately, even if such strategic analysis and sensing are often undertaken by certain executives, the nature of the slow and gradual progress and success of disruptive innovations often render it unbelievable that at one point in time, such innovations would turn to be disruptive. In most of the cases, most of the probable disruptive innovations are often not easily identifiable in their early stages. However, management and executives' trust and confidence in their business capabilities, experience gained over the years and indepth understanding of the industry dynamics often induce the tendencies for the executives to ignore capabilities of businesses that were identified to easily turn disruptive in their early stages. This affects proactive early interventions that could have been undertaken to curtail disrupters before they turn disruptive. It also implies to proactively curtail disruptive innovations; none of the emerging innovations that commence by targeting incumbents' lowend or new-market footholds must be taken for granted.

Quite often, most of the incumbents rely on momentum, tech-implementation, ecosystem, new technology and business model barriers as constrains that would curtail disrupters' creep into the upstream main market. However, as disrupters continue to undertake incremental improvements of their technologies and business models, risks often arise from the fact that in case of a breakthrough, incumbents that previously relied on such barriers become more vulnerable to complete disruptions. Even though continuous improvements would strengthen advantages derived from such barriers, most of the executives are often unwilling to commit unnecessarily large amount of resources on R\&D when the threats are not so much eminent. As all these cause complexities of discerning how to deal with disruptive innovations, such complexities are also further exacerbated by the unavailability of the appropriate business model that the contemporary business executives can replicate when dealing with disruptive innovations. Thus, to identify and mitigate disrupters' threats, it is argued in the context of the illustration in Figure 2 that it is critical for the executives to accomplish two sets of activities that encompass strategic analysis and sensing of disruptive innovations and countering disruptive innovations. 


\section{Boniface Okanga}

Countering Disruptive Innovations
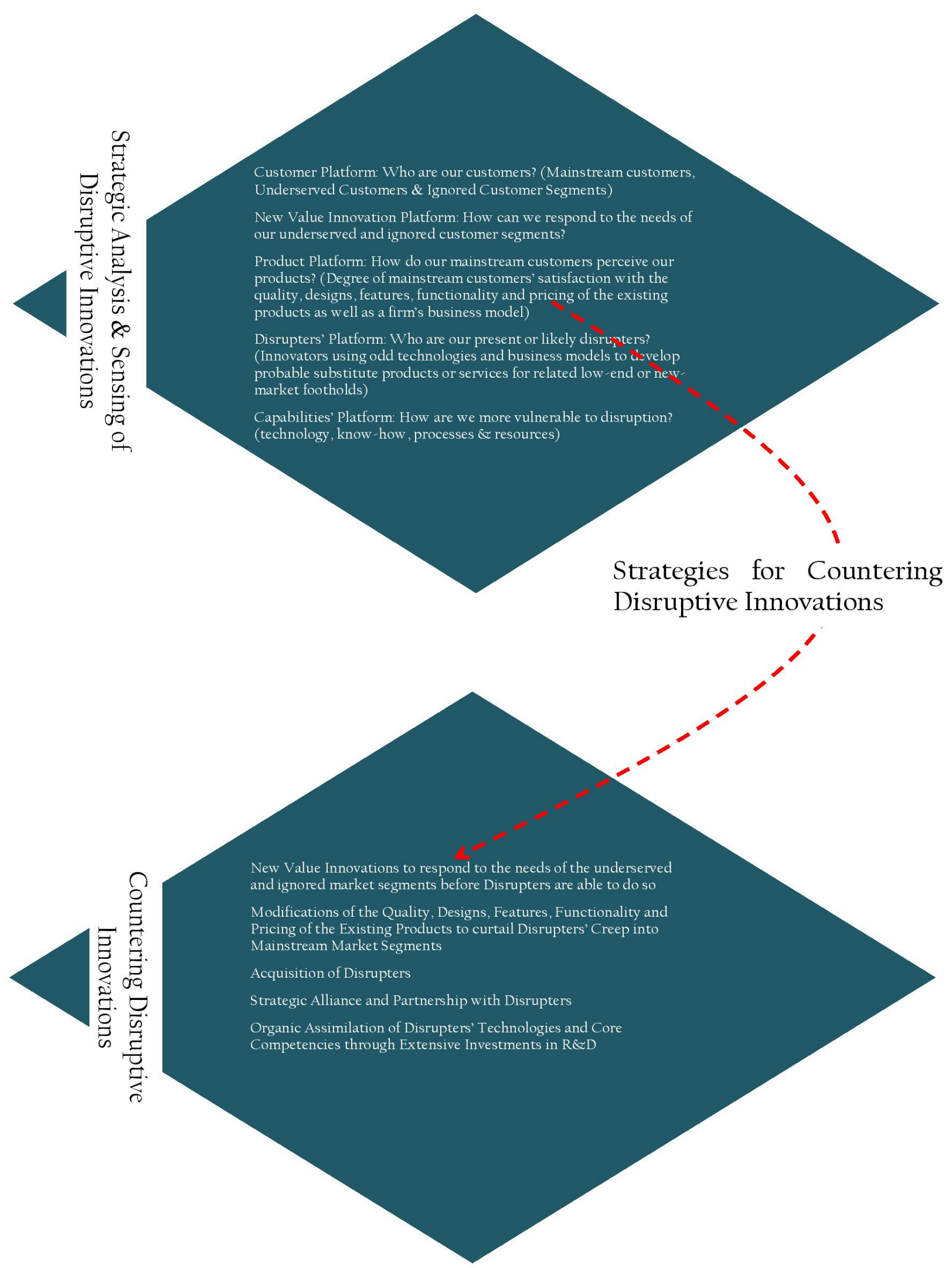

Figure 2: Generic Strategies for Countering Disruptive Innovations 


\section{Boniface Okanga \\ Countering Disruptive Innovations}

Strategic analysis and sensing of disruptive innovations will require the analysis of five business platforms that include customer, new value innovation, product, disrupters and capabilities' platforms. Since disrupters use low-end or new-market footholds, customer analysis is critical for a business to isolate and segment its mainstream customer segments from the underserved and ignored market segments. This evaluation aids the identification and blocking of the probable points of entrance that most new disruptive innovators are most likely to use.

To accomplish that, it is of essence for the business to use new value innovation platform to discern the initiatives that can be undertaken to leverage effective response to the demands and needs of the underserved and ignored customer segments. As such analysis is being undertaken, the assessment of product platform bolsters the evaluation of the degree of mainstream customers' satisfaction with the quality, designs, features, functionality and pricing of the existing products as well as a firm's business model. This is attributable to the fact that even if disruptive innovators do not use mainstream markets as points of entry, such analysis is still important for undertaking necessary product modifications to counter the disrupters that are already attempting to move from low-end or new-market footholds into a business' mainstream market segments.

To identify these probable disruptive innovators, the executives must undertake the analysis of the disrupters' platforms to identify the present and the most likely probable future disrupters. This can be accomplished by examining trends on the innovators that are already using odd technologies and business models to develop probable substitute products or services for related low-end or new-market footholds. Such analysis must be accompanied by the analysis of the capabilities' platform to diagnose the extent to which given the existing technologies, know-how, processes and resources, the disrupters are most likely to possess advantages that render a firm more vulnerable to disruptions.

However, since disruptive innovation is a process, it is still critical for the executives to undertake a combination of the required critical strategies to counter disruptive innovations even if a firm is found to possess superior capabilities than the identified likely disrupters. In the context of the illustration in Figure 2, countering disruptive innovations would require the executives to consider the conceptualisation and application of a combination of strategies encompassing new value innovations, product modifications, acquisition of the disrupters, strategic alliance and partnership with disrupters, and organic assimilation of disrupters' technologies and core competencies through extensive investments in R\&D.

Whereas new value innovations would enable a firm respond to the needs of the underserved and ignored market segments before disrupters are able to do so, modifications of the quality, designs, features, functionality and pricing of the existing products would enable a business curtail disrupters' creep into mainstream market segments. Even if these strategies aid thwarting of the identified disruptive innovations, it is still important that the executives undertake acquisition of disrupters and strategic alliance and partnership with disrupters to enable acquisition and assimilation of unique advantages derived from the disrupter's superior technologies, know-how and competencies. Such initiatives may be accompanied by organic assimilation of disrupters' technologies and core competencies through extensive investments in R\&D.

In otherwords, the concept of disruptive innovations is widely used in theories and among academics. This common usage of the phrase "disruptive innovations" is also accompanied by an avalanche of research on what disruptive innovations are or are not. However, research on how to deal with disruptive innovations remains largely limited. This has affected the development of theories as well as business models that the executives can replicate when dealing with disruptive innovations. Using the business model illustrated in Figure 2, this research attempted to address such a challenge. However, future research can 


\section{Boniface Okanga \\ Countering Disruptive Innovations}

still explore the key success factors that would influence effective application of such a model.

\section{Acknowledgements}

Special thanks to Professor Geoff Goldman, HoD of the Department of Business ManagementUniversity of Johannesburg and Professor Adri Drotskie, Director of the School of ManagementUniversity of Johannesburg for offering the intellectual support that rendered the successful accomplishment of this research.

\section{References}

- Assink, M. (2006). Inhibitors of Disruptive Innovation Capability: A Conceptual Model. European Journal of Innovation Management, 9(2):215-233. CrossRef

- Christensen, C.M., Raynor, M.E., and McDonald, R. (2015). What is Disruptive Innovations? Boston: Harvard Business Review.

- Christensen, C.M. (1995). Disruptive Technologies: Catching the Wave. Boston: Harvard Business Review.

- Christensen. C.M. (1997). Innovator's Dilemma. Boston: Harvard Business Review.

- Durantin, A., Fanmuy, G., Miet, S., and Pegon, V. (2017). Disruptive Innovation in Complex Systems. Cham: Springer. CrossRef

- Grant, R. (2010). Contemporary Strategic Management: Cases and Materials: New York: Pearson.

- Hekkert, M., and Simona, N.S. (2011). Understanding technological change explanation of different perspectives on innovation and technological change. Utrecht: Utrecht University Press.

- Kim, W.C., and Mauborgne, R. (2005). Blue ocean strategy: how to create uncontested market space and make the competition irrelevant. Boston: Harvard Business School Press.

- Raynor, M.E., and Mumtaz, A. (2013). Three Rules: How Exceptional Companies Think. New Yok: Penguin Books.

- Reeves, M., and Deimler, M. (2011). Adaptability: The new competitive advantage. Boston: Harvard Business School Review.

- Weeks, M. (2015). Is Disruption Theory Wearing New Clothes or Just Naked?Analysing recent Critiques of DisruptiveInnovation Theory. Innovation: Management, Policy \& Practice, 17(4):417-428. CrossRef

- Wessel, M., and Christensen, C.M. (2012). Surviving Disruption. Boston: Harvard Business Review.

- Yannopoulos, P. (2011). Defensive and offensive strategies for market success. International Journal of Business and Social Science 2(13):1-12. 